\title{
Molecular Bio-imprinting of Biocatalysts
}

\section{Israr Khan ${ }^{1}$ and Muhammad Waheed Akhtar ${ }^{1}$}

\section{Author 1:}

Israr Khan

\section{Affiliation:}

School of Biological Sciences, University of the Punjab, 54590, Lahore, Pakistan

\section{E-mail:}

Khanisrar07@gmail.com. israrks@live.com

Phone number: 00923349301550.

Author 2: Muhammad Waheed Akhtar

Affiliation:

School of Biological Sciences, University of the Punjab, 54590, Lahore, Pakistan

E-mail: mwapu@brain.net.pk

Phone number :92-42-99230-960

Fax No : - 92-42-99230-980 


\section{Abstract}

Energy conservation is the cry of the day. Attempts are made all over the world to occupy and use energy reserves. Increased industrialization and machanisation has led to the depletion of natural energy reserves. Its un avoidable to search for renewable sources of energy, may be not used now but can be used by the future generations. we are using the expertise of our ancestors. Thus exploiting the nature and newer techniques in this area would yield the best results. Bio-imprinting is one of those techniques whereby chemical modification of the E.coli culture medium is done in order to achieve highly expressed protein which can be stored in its highly active form in specific solvent.

Key Words: Biocatalyst, Bioimprinting, E. coli 


\title{
Introduction
}

\author{
C.thermocellum bglA gene for beta-D-glucosidase. \\ LOCUS X60268 $2021 \mathrm{bp}$ \\ SOURCE Clostridium thermocellum ATCC 27405 \\ CDS 361..1707
}

\section{ORIGIN}

gtcatcatatataaaagcgccagattagctccattgttgcttagctggaacctcgccctt ctctttaacctgcctaattccaccaaacattacccccatattatt taatatattatttg atatataattctttaatttatataattctttaattatctcaatctatttgctatttatc acattattcaatttaatacaaagaaaaaggctcataagctcaccataatataccataatc tttatgcttttcaggctattcatttttcgattaaaataaatagtatttatgtttcct ctaggttataattatattattacactgtttgcaaataatatcttaaagggtgtggtaaac atgtcaaagataactttcccaaaagatttcatatggggttctgcaacagcagcatatcag at tgaagg tgcatacaacgaagacggcaaaggtgaatctatatgggaccgtttttcccac acgccaggaaatatagcagacggacataccggcgatgttgcatgcgaccactatcatcgt tatgaagaagatatcaaaataatgaaagaaatcggtattaaatcatacaggttttccatc tcatggcccagaatctttcctgaaggaacaggtaaattaaatcaaaagggactggattt tacaaaaggctcacaaatctgcttctggaaaacggaattatgcctgcaatcactctttat cactgggacct tccccaaaagct tcaggataaaggcggatggaaaaaccgggacaccacc gattatttacagaatactctgaagtaatatttaaaatctcggagatatcgttccaata tggtttactcacaatgaacccggtgttgtttctttgcttggccactttttaggaattcat gcccctgggataaaagacctccgcact tcattggaagtctcgcacaatct tcttttgtcc cacggcaaggccgtgaaactgtttagagaaatgaatattgacgcccaaattggaatagct ctcaatttatcttaccattatcccgcatccgaaaaagctgaggatattgaagcagcggaa ttgtcatttctctggcgggaaggtggtatctggatcctgtgctaaaaggccggtatcct gaaaacgcattgaaactttataaaaagaagggtattgagctttctttccctgaagatgac ctgaaact tatcagtcagccaatagacttcatagcattcaacaattat tcttcggaattt ataaaatatgatccgtccagtgagtcaggtttttcacctgcaaactccatattagaaaag ttcgaaaaaacagatatgggctggatcatatatcctgaaggcttgtatgatctgcttatg ctccttgacagggattatggaaagccaaacattgttatcagcgaaaacggagccgccttc aaagatgaaataggtagcaacggaaagatagaagacacaaagagaatccaatatct taaa gat tatctgacccaggctcacagggcaattcaggacggtgtaaact taaaagcatactac ttgtggtcgcttttggacaactttgaatgggcttacgggtacaacaagagattcggaatc gttcacgtaaattttgatacgttggaaagaaaataaaggatagcggctactggtacaaa gaagtaatcaaaaacaacggttttaatcatacacagcaaacacggatacacatcataaa acataatgctaatgggcattcacgtccgaatgcccattagcattatatttcactctata actctaaaacaatatatttaccaatgttaatatgctcaaccctgtttttctcttaagc tcaatccagccatcccggcaatcact taccagtccagatacaccaacatgaaagagcata gaccacagcgaaagatacacaactattttct tccccacatat tgttttaaataaccatcc attaaaataccacccttcacattttctggatagttcacat

\section{Materials and Methods}

The bgIA gene was PCR amplified from Clostridium thermocellum ATCC 27405 strain. TA cloned in pTZ57RT vector and transformed in DH5-alpha. Blue/White screening done to know the positive colonies. White colonies picked up and and colony PCR done to amplify the bgIA gene. Plasmid islolated and amplified 
for the presence of the gene. Positive colonies were replica spotted on L.B ampicillin.

The purified gene was cloned in pET28a vector and transformation done in BL21C(+). Positive colonies were replica spotted on L.B kanamycine. The spotted colonies picked up and cultured on L.B and M9NG medium and induced with IPTG and Lactose. Expression of the protein checked on 12\% SDS-PAGE.

The protein was purified, lyophilized and stored in different Inorganic and organic solutions like Na-P buffer, PBS, isopropanaol and Glycerol.

The theoratical PCR product with forward and reverse primers: ctcc atggtgtcaaagataaccttcccaaaagatttcatatggggttctgcaacagcagcatat IF 1-60 cagattgaaggtgcatacaacgaagacggcaaaggtgaatctatatgggaccgtttttcc cacacgccaggaaatatagcagacggacataccggcgatgttgcatgcgaccactatcat cgttatgaagaagatatcaaaataatgaaagaaatcggtattaaatcatacaggttttcc atctcatggcccagaatctttcctgaaggaacaggtaaat taaatcaaaagggactggat ttttacaaaaggctcacaaatctgcttctggaaaacggaat tatgcctgcaatcactctt tatcactgggacct tccccaaaagct tcaggataaaggcggatggaaaaaccgggacacc accgattatttacagaatactctgaag taatatt taaaaatctcggagatatcgttcca atatggtttactcacaatgaacccggtgttgtttcttgcttggccacttttaggaatt catgcccctgggataaaagacctccgcacttcattggaagtctcgcacaatcttcttttg tcccacggcaaggccgtgaaactgtttagagaaatgaatattgacgcccaaat tggaata gctctcaatt tatcttaccattatcccgcatccgaaaaagctgaggatat tgaagcagcg gaattgtcattttctctggcgggaaggtggtatctggatcctgtgctaaaaggccggtat cctgaaaacgcattgaaact tataaaaagaagggtattgagctttctttccctgaagat gacctgaaact tatcagtcagccaatagacttcatagcattcaacaattattcttcggaa tt tataaaatatgatccgtccagtgagtcaggttttcacctgcaaactccatat tagaa aagttcgaaaaaacagatatgggctggatcatatatcctgaaggcttgtatgatctgctt atgctccttgacagggat tatggaaagccaaacattgttatcagcgaaaacggagccgcc t tcaaagatgaaataggtagcaacggaaagatagaagacacaaagagaatccaatatct $t$ aaagat tatctgacccaggctcacagggcaat tcaggacggtgtaaact taaaagcatac tacttgtggtcgcttttggacaactttgaatgggcttacgggtacaacaagagattcgga atcgttcacgtaaattt tgatacgttggaaagaaaataaaggatagcggctactggtac 1340 aaagaagtaatcaaaaacaacggttttaatcatacacagcaaacacggatacacatcat 1400 gagctcag

\section{Results and discussions}

Recent efforts have been directed towards molecular cloning, sequencing, mutagenesis and crystallography of the enzyme. The sources and properties of recombinant beta glucosidase, the classification schemes based on similarity at the structural and molecular level, elucidation of structure function relationship, directed evolution of existing enzyme toward enhanced thermostability, substrate range, 
biosynthetic properties and application. (Khan and Akhtar., 2010). Protein engineering is the novel field which has wide applications from pharmaceutics, industry, commercial, laundry and research. It may apply rational design or non rational design or both. Site directed mutagenesis is a classical approach involving the protein folding principles and as such different techniques involving multidisciplinary research and broad knowledge is required involving biocomputing of complex data obtained from various sequencing projects and prediction of the future protein structure either chemically or genetically modified. Non rational mutagenesis or directed evolution involves random mutations in the gene encoding protein or shuffling the genes encoding different domains producing a random set of numerous large libraries of mutant proteins, using advanced technology the desired protein can be selected but the exact structure or changes may remain unnoticed (Khan and Akhtar., 2010). Sodium dodecyl sulfate (SDS) is an anionic detergent which denatures proteins by wrapping around the polypeptide backbone and SDS binds to proteins fairly specifically in a mass ratio of $1.4: 1$. In so doing SDS confers a negative charge to the polypeptide in proportion to its length i.e the denatured polypeptides become rods of negative charge with equal charge or charge densities per unit length. In denaturing SDS-PAGE separations therefore, migration is determined not by intrinsic electrical charge of the polypeptide, but by molecular weight. 12\% SDS-PAGE of both Lactose induced (fig. 3) and IPTG induced (fig. 4) showed an estimated protein of $51 \mathrm{Kda}$. Besides developments in molecular biology and biochemistry, biocomputing and methods for the determination of 3D structures, are indispensable tools in this field. Since the three-dimensional structure of the 
protein determines its biological function, it becomes important to have available a high resolution 3D structure, especially when the protein engineer aims to redesigning the protein function. Often enzymes exposed to particular $\mathrm{pH}$ or ligands before liophylization and subsequent dissolution in organic solvents, prove to be able to retain specific properties, i.e affinity for the ligand, a property referred to as "memory". Based on this observation, a strategy for the activation of proteins has been developed called "Molecular Bio-imprinting" and can be adapted to particular conditions where the reaction of interest has to take place. Thus Bio-imprinting of is demonstrated to be an easy, feasible and effective method for obtaining overexpressed and highly active enzymes in organic solvents.

\section{Acknowledgement:}

We are Very Much thankful to Prof. Dr Muhammad Akhtar, Director General, school of Biological sciences, University of the Punjab, Pakistan and Prof. Dr. Atta UI rehman, ex chairman Higher Education Commission Pakistan (HEC) for their valuable contribution. 


\section{Literature cited:}

1. Jarvis M. 2003. Cellulose stacks up. Science. 426:611 - 612 .

2. Lynd LR, Weimer PJ, van Zyl WH, Pretorius IS. 2002. Microbial cellulose utilization: fundamentals and biotechnology. Microbiol Mol Biol Rev. 66:506577.

3. Gardner KH, Blackwell J. 1974b. The hydrogen bonding in cellulose. Biochim Biophys Acta 343:232-237.

4. Spezio,M.,D.B Wilson, and P.A Karplus. 1993. "Crystal structure of the catalytic domain of thermophilic endocellulase" Biochem. J., 222:729-736.

5. Pommier J. C.; Goma G.; Fuentes J. L.; Rousset C.;Jokinen O. Tappi 1990, 73, 197-202.

6. Wildman, K.H. and D. Kern.,Dynamic personalities of Proteins.2007. Nature/vol450/doi:10.1038/nature06522

7. Khan I, Shuaib M, Sikandar, Zia M A, and Akbar H. 2010. A Micropropagation, Molecular and Physiochemical Analysis of Canola Brassica napus L. From Seeds. Available from Nature Precedings http://hdl.handle.net/10101/npre.2010.4781.2

8. Khan I and Akhtar M W. 2011. Different Approaches For Protein Engineering In Industrial Biotechnology. Nature Precedings

http://hdl.handle.net/10101/npre.2011.5601.1 
Figures and Tables legends

Figure 1: Colonies on X-Gal plate screened by Colony PCR for bgIA/pTZR and loaded on $0.8 \%$ Agarose gel. showing. $\mathrm{M}$; VC $1 \mathrm{~Kb}$ protein marker. Purified gene cleaned bglA $1.4 \mathrm{~Kb}$ (Lane 1). Positive colonies/white ( Lane 4 and Lane 5). Negative colonies/blue (Lane 2 and Lane 3)

Figure 2: $12 \%$ SDS-gel of total cell protein of E. coli BL21(DE3) cells expressing $\beta$ glucosidase ( $\mathrm{pET} 28 \mathrm{a}-\mathrm{BGIA-1)}$ after induction Lactose concentrations. M; vivantis protein marker. Induced samples and Uninduced samples.

Figure 1.

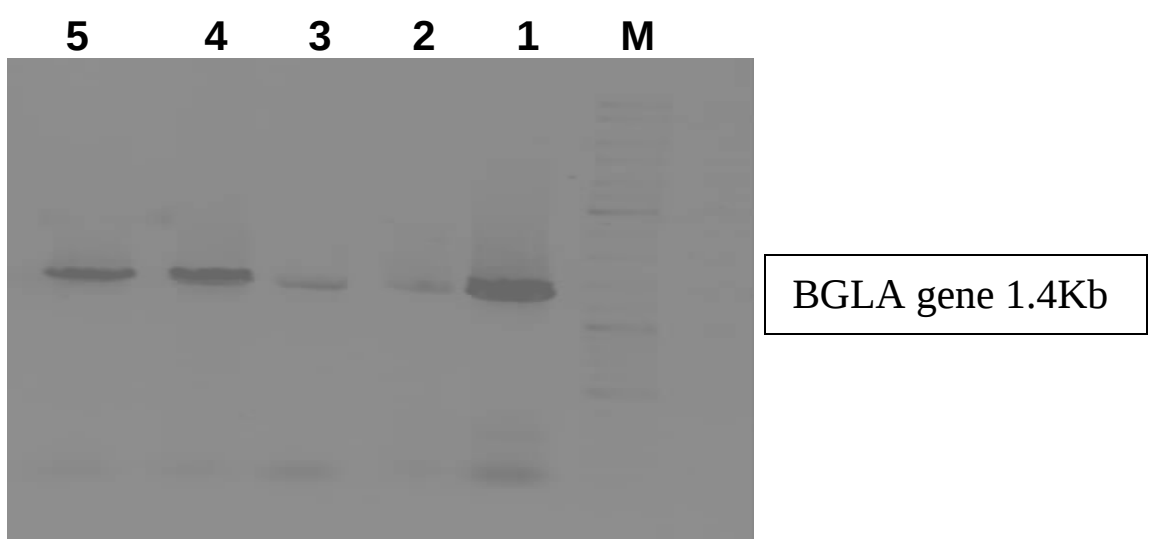

Figure 2.

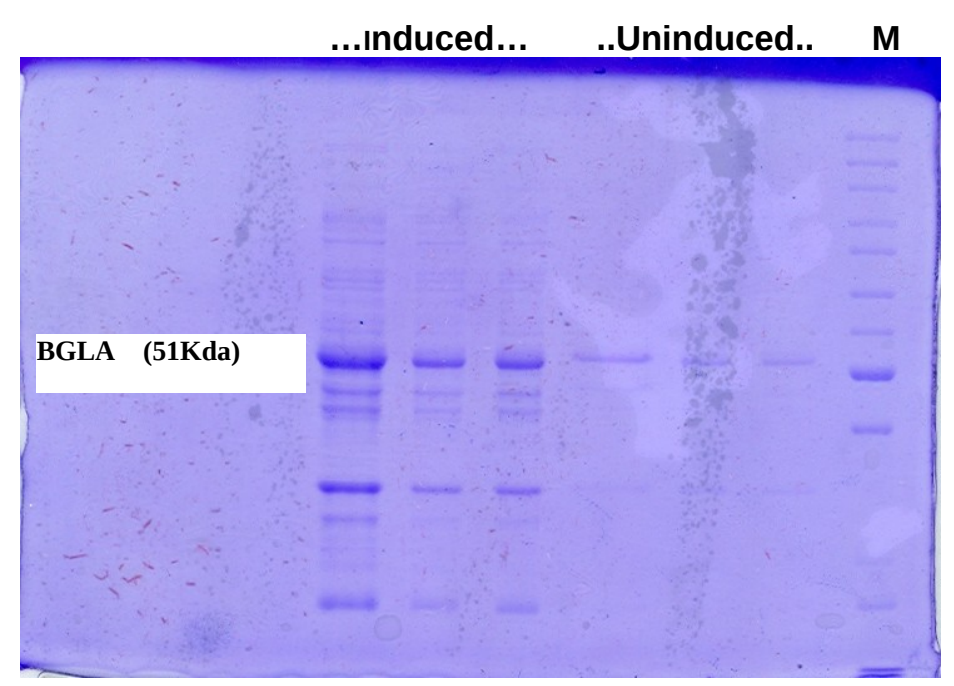

